\title{
Quantitative 3D electromagnetic field determination of 1D nanostructures from single projection
}

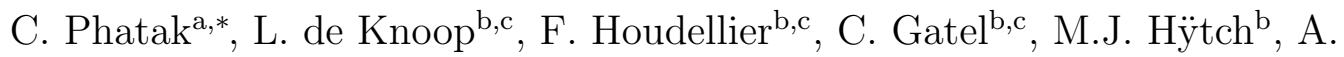 \\ Masseboeuf ${ }^{b}$ \\ ${ }^{a}$ Materials Science Division, Argonne National Laboratory, Argonne, IL 60439, USA. \\ ${ }^{b}$ CEMES-CNRS, 29 rue Jeanne Marvig, F-31055 Toulouse, France. \\ ${ }^{c}$ Université Paul Sabatier, F-31000 Toulouse, France.
}

\section{Abstract}

One-dimensional (1D) nanostructures have been regarded as the most promising building blocks for nanoelectronics and nanocomposite material systems as well as for alternative energy applications. Although they result in confinement of a material, their properties and interactions with other nanostructures are still very much three-dimensional (3D) in nature. In this work, we present a novel method for quantitative determination of the 3D electromagnetic fields in and around 1D nanostructures using a single electron wave phase image, thereby eliminating the cumbersome acquisition of tomographic data. Using symmetry arguments, we have reconstructed the 3D magnetic field of a nickel nanowire as well as the 3D electric field around a carbon nanotube field emitter, from one single projection. The accuracy of quantitative values determined here is shown to be a better fit to the physics at play than the value obtained by conventional analysis. Moreover the $3 \mathrm{D}$ reconstructions can then directly be visualized and used in the design of functional 3D

\footnotetext{
* Corresponding author

Email address: cd@anl.gov (C. Phatak)
} 
architectures built using 1D nanostructures.

Keywords: 3D electromagnetic fields, 1D nanostructures, phase reconstruction, transmission electron microscopy

\section{Introduction}

2

Nanoscale structures, such as ultra-thin films, nanoparticles, and nanowires, have been intensively investigated over the past decade as a result of their novel size-dependant behavior. Amongst them, one dimensional (1D) nanostructures such as nanowires and nanotubes are of significant importance as they exhibit molecular scale properties and behavior such as density of states singularities, and emergent charge transport properties [1, 2, 3]. These structures also have direct applications as functional nanostructures in nanoelectronics and nanophotonics. The recent progress in fabrication methods have enabled 1D nanostructures to be made from a wide variety of materials including ferromagnetic [4], ferroelectric [5], thermoelectric [6] and semiconductor [7]. In all these 1D nanostructures, it is necessary to quantitatively measure the local properties so that their behavior can be better understood. Moreover, enhancement of some properties such as local electromagnetic fields in 1D nanostructures needs to be quantified, so that it can be controlled for required applications.

In this work we focus on two particular 1D nanostructures: (1) ferromagnetic nanowires, and (2) electrostatically charged carbon nanotips. Ferromagnetic nanowires form an integral part of existing and novel applications in magnetic storage [8] and sensing [9]. Magnetic nanowires with circular cross-section, are of utmost importance from theoretical and technological 
aspects. Their high aspect ratio and cylindrical symmetry leads to novel domain wall behavior such as suppression of Walker breakdown, which is of interest in magnetic logic circuits and domain wall memory [10, 11]. They also have potential applications in single cell bio-sensing $[12,13]$ as well as in nanocomposites for exchange spring magnets [14]. They can be fabricated using a variety of methods such as lithography based templating [15], wet chemical methods and electro deposition [16]. 1D carbon-based nanostructures such as carbon nanotubes are amongst the best candidates for field emission displays [17] and new high-brightness electron sources [18]. Since their discovery by Iijima [19], carbon nanotubes have been extensively studied and are now used in a wide variety applications [20, 21]. One such application is field emitters because they provide a unique combination of high conductivity, high aspect ratio and robustness [22]. A carbon cone nanotip (CCnT) was recently successfully used in a high-voltage electron gun [23].

The confinement effects in 1D nanostructures can alter their properties and subsequently their behavior significantly. Hence it is necessary to understand the strong effect of their size on their three-dimensional (3D) properties such as the magnetic and electric fields associated with nanowires and nanotubes completely before they can be used in applications. There are a variety of methods to study electromagnetic fields at the nanometer length scale such as scanning tunneling microscopy [4], scanning electron microscopy with polarization analysis (SEMPA) [24], and transmission electron microscopy (TEM) $[25,26,27,28]$. TEM offers the best approach because it can be used to determine their physical/crystal structure, composition as well as to image the electromagnetic field at a high spatial resolution. In particular for 
Lorentz TEM, the electromagnetic information of the sample is encoded in the phase shift of the electron wave, $\phi$, and can be written as a combination of projections of the 3D magnetic vector potential, A, and 3D electrostatic potential, $V$, given as [29]:

$$
\begin{aligned}
\phi(x, y) & =\phi_{e}(x, y)-\phi_{m}(x, y) \\
& =C_{E} \int_{-\infty}^{+\infty} V(x, y, z) \mathrm{d} z-C_{B} \int_{-\infty}^{+\infty} A_{z}(x, y, z) \mathrm{d} z
\end{aligned}
$$

where $\phi_{e}$ is the electrostatic phase shift, $\phi_{m}$ is the magnetic phase shift, $C_{E}=\pi / E \lambda, E$, the relativistic electron scattering potential and $\lambda$, the wavelength of electrons, $C_{B}=\pi / \Phi_{0}, \Phi_{0}$, the magnetic flux quantum and the direction of propagation of the electrons is assumed to be along $z$. The phase shift can be recovered experimentally using various techniques such as transport-of-intensity based methods or off-axis electron holography. However, from the equations it can be seen that the recovered phase shifts are an integration of the signal over the electron path that yield only projected 2D information. This means that the information from the sample itself is integrated along with the stray field, thereby leading to inaccurate measurements of electromagnetic fields. There are currently very few methods, which have the capability to visualize the complete $3 \mathrm{D}$ vector fields associated with nanowires. Nominally, determining the 3D magnetic field requires recording a series of phase shift images as the sample is tilted about its axis. This becomes a tedious and cumbersome process with various sources of error or requires specialized electron microscopes [30,31]. In a 1D nanostructure such as a nanocylinder that is uniformly magnetized along its long axis, the magnetic field possesses cylindrical symmetry with respect to the long axis. 
In cases, where the 1D nanostructure does not possess cylindrical symmetry, the usual tilt based tomography must be performed to reconstruct the 3D magnetic field [32]. Similarly, carbon nanotubes under applied bias exhibit a cylindrically symmetric potential and electric field. Here we show that the cylindrical symmetry can be exploited to enhance the quantification of electromagnetic properties by recovering the entire 3D vectorial field in and around nanowires and nanotubes from a single image.

\section{3D magnetic field}

The magnetic information from the sample is contained in the second term of equation (1), $\phi_{m}$, which can be separated using time reversal symmetry, i.e. by recording the phase shift additionally with the sample turned over by $180^{\circ}$ and computing the difference between the two phase shifts [33]. Taking the derivative of the magnetic phase shift with respect to $x$, and using the relation between vector potential and magnetic induction $(\mathbf{B}=\nabla \times \mathbf{A})$ gives:

$$
\frac{\partial}{\partial x} \phi(x, y)=C_{B} \int_{-\infty}^{\infty} B_{y}(x, y, z) \mathrm{d} z
$$

This equation correlates the projection of one component of the magnetic induction with the magnetic phase shift image. When there is a cylindricallysymmetric magnetic induction present, using the inverse Abel transform [34], the $y$ component of the magnetic induction, $B_{y}$, can be reconstructed from the phase shift image as:

$$
B_{y}(\rho, y)=-\frac{1}{C_{B} \pi} \int_{\rho}^{\infty} \frac{\partial^{2} \phi(x, y)}{\partial x^{2}} \frac{\mathrm{d} x}{\sqrt{x^{2}-\rho^{2}}}
$$


where $\rho^{2}=x^{2}+z^{2}$. A detailed derivation of the inverse Abel transform is presented in the supporting information. It should be noted that using the above equation provides the $y$ component of all magnetization vectors in 3D. Furthermore, using the divergenceless condition $\nabla \cdot \mathbf{B}=0$ in cylindrical coordinates, $B_{\rho}$ can be calculated as:

$$
B_{\rho}(\rho, y)=-\frac{1}{\rho} \int_{\rho}^{\infty} \rho^{\prime} \frac{\partial B_{y}\left(\rho^{\prime}, y\right)}{\partial y} \mathrm{~d} \rho^{\prime}
$$

the $x$ and $z$ components of the magnetic induction being obtained as $\left(B_{x}, B_{z}\right)=$ $\left(B_{\rho} \cos \theta, B_{\rho} \sin \theta\right)$.

The numerical implementation of the reconstruction procedure was done using the Interactive Data Language (IDL) and was tested by reconstructing the magnetic induction of a uniformly magnetized spherical nanoparticle as the analytical expression for this case is well known. The simulations were performed using a $256 \times 256$ pixel grid with a resolution of $1 \mathrm{~nm}$ per pixel. The magnetic induction of such a sphere has cylindrical symmetry about the axis of the magnetization vector [35], which here is assumed to be lying in the $x-y$ plane. The computations were performed for a uniformly magnetized sphere of radius, $R=16 \mathrm{~nm}, B_{0}=1.0 \mathrm{~T}$, and magnetization unit vector given by $[0,1,0]$. The magnetic phase shift of such a sphere was numerically computed using the analytical expression given in the paper by De Graef et. al. [36]. Figure 1(a) shows the simulated magnetic phase shift image of the spherical nanoparticle. Figure 1(b) shows the derivative of the magnetic phase shift with respect to the $x$ axis (horizontal axis of the image) required for reconstruction (equation (2)). The symmetry about the $y$ axis of the image (vertical axis) in the derivative image can be clearly observed. This 
image was then used for numerical implementation of equation (3) to reconstruct the complete 3D magnetic induction inside and outside the spherical nanoparticle shown in Figure 1(c). The inverse Abel transform was computed using the modified Fourier-Hankel algorithm described by S. Ma et al. [37]. The $B_{\rho}$ component was then calculated by numerically implementing equation (4) in polar coordinates. The numerical integration was performed using the 5-point Newton-Cotes integration formula. The vector quantities, $B_{y}$ and $B_{\rho}$ as well as the scalar potential, $V$ were then interpolated from polar coordinates to Cartesian coordinates. The color of the arrows indicates the magnitude of the $y$ component of the magnetic induction with respect to the color bar, in units of $\mathrm{T}$. The main advantage of this method, as illustrated in Figure 1, is that it can be directly used to quantitatively map the 3D magnetic induction, which leads to a better quantification of magnetic induction within the nanoparticle by eliminating the effect of surrounding stray fields. This can yield a more thorough understanding of the strength of magnetic interactions between spherical magnetic nanoparticles that can be useful for the self-assembly process. Additional comparisons as well as systematic errors analysis can be found in the supplementary material.

\section{3D electric field}

The electrostatic information from the sample is similarly contained in the first term of equation (1), $\phi_{e}$. This term contains the contribution due to the charge density in the sample resulting from the applied bias as well as the mean inner potential (MIP) of the sample. The MIP can be assumed to be constant inside the sample, and hence its contribution to the electrostatic 
phase shift depends only on the sample's thickness variation. This contribution can be eliminated by computing the difference between phase shifts of unbiased and biased samples. Resulting phase shift, $\phi_{e, q}$, due to only the charge density can then be written as:

$$
\phi_{e, q}(x, y)=C_{E} \int_{-\infty}^{\infty} V_{q}(x, y, z) \mathrm{d} z
$$

where $V_{q}$ represents the potential due to the charge density in the biased sample. For a sample with cylindrically-symmetric potential, and assuming that the sample is oriented such that the axis of symmetry is along the $y$ axis, inverse Abel transform can be used to reconstruct the 3D electrostatic potential as:

$$
V_{q}(\rho, y)=-\frac{1}{C_{E} \pi} \int_{\rho}^{\infty}\left(\frac{\partial \phi_{e, q}(x, y)}{\partial x}\right) \frac{\mathrm{d} x}{\sqrt{x^{2}-\rho^{2}}}
$$

The electric field of the sample can then be computed numerically using the relation $\mathbf{E}(\mathbf{r})=-\vec{\nabla} V$, where $\vec{\nabla}$ is the gradient operator in 3D.

\section{Results}

Magnetic nanowire. Magnetic nanowires with circular cross section which are uniformly magnetized along their long axis also posses cylindrically symmetric magnetic induction. Similar to the case of the uniformly magnetized nanosphere, it is possible to compute the complete 3D magnetic field of such a nanowire using a single phase image. Experiments were performed on a polycrystalline nickel $(\mathrm{Ni})$ nanowire of $75 \mathrm{~nm}$ diameter [16]. The nickel nanowires were grown by electro deposition in commercial $6 \mu \mathrm{m}$ thick 
polycarbonate membranes. The membranes were then dissolved in $\mathrm{CH}_{2} \mathrm{Cl}_{2}$, and the Ni nanowires were recovered on a carbon foil for TEM imaging. Only the region near the extremity of the nanowire was used for reconstruction such that information about the magnetic field within the nanowire can be acquired along with the stray fields.

Figure 2(a) and (b) shows the experimental electrostatic and magnetic phase shifts of the nanowire. The phase shift was recovered using off-axis electron holography in a FEI Tecnai F20 TEM operating at $200 \mathrm{kV}$ using the first transfer lens of the Cs-corrector as a Lorentz lens. The magnetic phase shift was obtained from the Ni nanowire by recording the phase shift images with the sample as-is and rotating it by $180^{\circ}$ about its axis and then subtracting the two phase shifts. The mean inner potential (MIP) contribution to the phase shift gives an overview of the shape of the wire. Moreover, a weak electric field in vacuum can be seen due to charging effect on the nanowire giving us high confidence into the separation of the two phase shifts. The magnetic phase image was then used to perform the numerical reconstruction of the 3D magnetic field. The experimental reconstructions were performed on a $256^{3}$ grid. Figure 2(c) shows the conventional approach of estimating magnetic induction in the nanowire using the relation, $B_{\text {proj }}=$ $\nabla \phi_{m} / C_{B} t$, computed from the gradient of the phase shift and dividing by the thickness, $t$ of the sample, derived from the MIP. With the same phase data, now we can reconstruct the entire 3D magnetic field. A vectorial view of the reconstruction can thus be displayed(Figure 2(e)) to emphasis the vectorial 3D nature of the new dataset. Moreover we can visualize and measure the magnetic induction in the $x-y$ plane in the middle of the nanowire as shown 
in Figure 2(d).

Such slicing capability is the main advantage of our method as we can obtain more precise estimate of the magnetic saturation in the nanowire as shown in Figure 3. The conventional integrative approach (Figure 2(c)) typically does not account for the integration of the stray fields outside the nanowire leading to an inaccurate quantification of the magnetic saturation of the material. This is schematically shown in Figure 3(a) where the stray fields are shown in the plane above the nanowire, the true magnetic induction of the sample is measured in the mid-plane of the nanowire, and the measured magnetic induction in the projected plane is the integration of these values. The $\mu_{0} \cdot M_{s}$ is now measured as $0.7 \pm 0.1 \mathrm{~T}$ as compared to the $0.5 \pm 0.1 \mathrm{~T}$ value obtained from the conventional phase gradient method as shown by the lineplots in Figure 3(b). It worth noting that this new experimental value for magnetic saturation of $\mathrm{Ni}$ was the one that was found to best fit micromagnetic simulations performed for domain wall studies in Ni nanowires [16]. The underestimation due to stray field contribution is eliminated in our measurements. It is however limited to the extent of the reconstruction volume. The strong decrease of the field away from the nanowire $\left(1 / r^{3}\right)$ versus the size of the reconstruction volume $\left(\sim 286 \mathrm{~nm}^{3}\right)$ is sufficient to assume the complete removal of the stray field versus our sensitivity. The only remaining source of error in the quantification comes from the perturbed reference wave used for performing holographic phase reconstruction. The experimental induction map suffers slightly from a noisy phase reconstruction which is caused due to the strong diffraction contrast from the polycrystalline Ni nanowire. A Savitzky-Golay differentiation filter [38] was used to minimize 
the effect of this noise.

Charged Nanotube There is intense interest in exploring carbon nanostructures for high efficiency field emitter applications: in particular carbon nanotubes and their derivatives. When an electrical bias is applied to a carbon nanotube, charge builds up on the surface, which is proportional to the applied bias until field emission occurs. The cylindrical geometry of the nanotubes results in a cylindrically-symmetric charge distribution and potential. It is possible to measure the effect of such a potential by measuring the resulting phase shift using off-axis electron holography. Here we have used the single image method developed in this work to determine the 3D potential around the charge distribution as well as to obtain the 3D electric field around the carbon nanotube.

Experiments were performed on carbon cone nanotip (CCnT) using an in-situ TEM holder (Nanofactory Instruments), in which the CCnT was exposed to an applied bias and the phase shift was measured using off-axis holography [39] (Figure 4(a)). The carbon cone nanotips (CCnTs) were prepared by deposition of pyrolitic carbon onto a multi-wall carbon nanotube. The CCnTs were then mounted on a tungsten tip using a dual Focused Ion Beam/FEG Scanning Electron Microscope (FIB/SEM). Figure 4(b) shows the phase image of the CCnT under an applied bias of $60 \mathrm{~V}$. The contours are drawn every $\pi$ radians. Figure $4(\mathrm{c})$ shows the $3 \mathrm{D}$ distribution of the reconstructed potential along with contours drawn every $10 \mathrm{~V}$ associated with the CCnT (shown in gray). The color bar shows the magnitude of the potential in volts. The reconstructed $3 \mathrm{D}$ potential from the CCnT was then used to compute the 3D electric field as shown in Figure 5. 
Figure 5(a) shows the conventional approach for estimating the electric field from the phase shift, similar to that for magnetic induction by computing the gradient of the phase shift. This method again represents an integrated measurement of the electric field that leads in that case to an overestimation of the measurement due to stray fields. Figure 5(b) shows the reconstructed electric field in the $x-y$ plane in the middle of the CCnT obtained using our method. Figure 5(c) shows the quantitative comparison of the electric field as measured from the projection and in the middle of the $\mathrm{CCnT}$, with the vertical dashed line showing the edge of the CCnT. Similar to the magnetic saturation measurement, here too, we can see that we have more accurate estimate of the electric field at the apex of the $\mathrm{CCnT}$ since we are removing the effect of field outside the CCnT. Figure 5(d) shows the $3 \mathrm{D}$ vector field plot of the electric field in and around the $\mathrm{CCnT}$, with the arrows colored according to the magnitude of the electric field in units of $\mathrm{V} / \mathrm{nm}$ as shown by the colorbar. The quantitative analysis of such a biased $\mathrm{CCnT}$ field emission process is rather easy as a direct local electric field can be instantly measured with better accuracy. We were thus able to measure an electric field of $2 \pm 0.5 \mathrm{~V} \cdot \mathrm{nm}^{-1}$ at the extremity of the CCnT. Accurate measurement of this value is of importance as it enables the estimation of the field enhancement factor [40] that is used for Fowler-Nordheim plot analysis and work function estimation of carbon nanotubes. Knowing this value as well as the applied electrical bias and anode- $\mathrm{CCnT}$ distance, one can estimate a field enhancement value of $\gamma=23 \pm 6$ which is consistent with the one estimated using finite element modelling [39]. The estimation here is once again only limited by the disturbed reference wave that causes a lowering of 
the measured electric potential in the image.

\section{Discussion}

The primary requirement for this method is that the field should be cylindrically symmetric. This necessitates that the axis of symmetry in the phase image is accurately determined prior to the 3D reconstruction of the field. However, experimental limitations, such as low spatial resolution or a magnetization distribution that is not completely symmetric, may prevent the accurate determination of the axis of symmetry. Furthermore, other experimental limitations such as the biprism orientation being not exactly perpendicular to the long axis of the 1D nanostructure can also introduce error in preserving cylindrical symmetry in the reconstruction. It is also true for the determination of the 3D electric potential and field. For example, the magnetization of a uniformly magnetized nanosphere can have a $z$-component but depending on the direction of projection, the phase shift may appear to be cylindrically symmetric. Another possibility is that the magnetization can be in a ' $\mathrm{S}$ ' or ' $\mathrm{C}$ ' state such that at the edge of the particle, it rotates in plane, thus leading to incomplete cylindrical symmetry. In order to assess the effect of variation in symmetry and possible errors in determining the symmetric axis, the magnetization of the spherical nanoparticle was rotated by an angle $\theta$ varying from $0^{\circ}$ to $20^{\circ}$ about the $y$ axis in the $x-y$ plane. The phase shift was then computed for the corresponding magnetization. However, while implementing and performing the 3D reconstruction of the magnetic induction, the symmetric axis was assumed to be along $y$ axis. The 3D magnetic induction was then reconstructed using the single 
image method and compared with the true 3D magnetic induction calculated analytically. Of particular importance and relevance is the axial field magnitude $\left(B_{y}\right)$ that was compared numerically: for example the theoretical and reconstructed value of axial field at the tip of the sphere is $0.66 \mathrm{~T}$ and $0.63 \mathrm{~T}$ for $\theta=0$ and decreases to $0.62 \mathrm{~T}$ and $0.59 \mathrm{~T}$ for $\theta=20^{\circ}$ respectively. This corresponds to only about 5\% error. Similarly the error in the angle of the magnetization vector was also compared. The comparison showed that the error in determination of the angle of the magnetization vector is smaller inside the nanosphere than outside. This can be related to the fact that outside the nanosphere, the magnetization vector changes direction rapidly as compared to the magnetization vector inside the sphere. Additional details about the calculation of errors and a rigorous quantitative comparison of errors is given in the supporting information. The final source of error in the quantitative measurement of the electromagnetic fields using our method is due to the perturbed reference wave. However, it has been shown previously that the reference wave perturbation at the length scale of the overlap area is not significant with error below 10\%.[39, 41].This demonstrates that although the condition of cylindrical symmetry is a fairly strict criterion for applicability of this method, for experimental purposes, this method can still reliably give quantitative values of magnetic induction or electric field in and around the nanostructures in 3D.

Finally, other approaches that quantitatively measure electromagnetic fields using phase reconstructed electron microscopy apart from tomographic methods, rely on building models of the sample either through finite element simulations or analytical models and then compare the experimental 
2D measurements with the results obtained from the models. Recent work by Beleggia et. al. have used this approach to show that using off-axis holography combined with theoretical modeling, it is possible to quantitatively measure the electric field around the tip of a biased needle and accurately take into account the shape of the tip [42]. However, the method presented in this work is unique in that it relies on experimental measurements to directly reconstruct the 3D electromagnetic fields.

\section{Conclusion}

Here we have successfully demonstrated that by exploiting the cylindrical symmetry of 1D nanostructures and the associated electromagnetic fields, it is indeed possible to reconstruct the entire 3D electromagnetic field from a single TEM phase image. This significantly reduces the time required to acquire multiple tilt series images as well as reducing the electron dose to the sample that is conventionally required for 3D reconstruction. The method was numerically implemented to reconstruct the 3D magnetic induction in and around a ferromagnetic nickel nanowire as well as the electric field in and around a carbon cone nanotip under applied bias. In both the cases, we have shown that using the information obtained in $3 \mathrm{D}$, we can get more precise measurement of relevant physical quantities such as magnetic saturation, or field enhancement factors as compared to conventional approaches. This work opens new paths for quantitative analysis of the 3D electromagnetic interactions for technologically important one-dimensional nanostructures. 
325

326

\section{Acknowledgments}

Work by C.P was supported by the U.S. Department of Energy (DOE), Office of Science, Materials Sciences and Engineering Division. A.M. and C.G are supported by the French national project EMMA (ANR12 BS10 013 01). A.M., C.G., L.K \& M.H. acknowledge the European Union under the Seventh Framework Programme under a contract for an Integrated Infrastructure Initiative Reference 312483-ESTEEM2 as well as the French National Research Agency under the "Investissement d'Avenir" program reference No. ANR-10-EQPX-38-01 and the "Conseil Regional Midi-Pyrénées" and the European FEDER for financial support within the CPER program. A.M. and C.P. acknowledge support by Université Paul Sabatier (APC programme) for funding the venue of C.P. at CEMES laboratory. 
[1] C. M. Lieber, One-dimensional nanostructures: Chemistry, physics \& applications, Solid State Communications 107 (11) (1998) 607-616. doi:10.1016/S0038-1098(98)00209-9.

URL http://www.sciencedirect.com/science/article/pii/S0038109898002099

[2] Y. Xia, P. Yang, Y. Sun, Y. Wu, B. Mayers, B. Gates, Y. Yin, F. Kim, H. Yan, One-dimensional nanostructures: Synthesis, characterization, and applications, Advanced Materials 15 (5) (2003) 353-389. doi:10.1002/adma.200390087.

URL http://onlinelibrary.wiley.com/doi/10.1002/adma.200390087/abstract

[3] S. V. Kuchibhatla, A. Karakoti, D. Bera, S. Seal, One dimensional nanostructured materials, Progress in Materials Science 52 (5) (2007) 699-913. doi:10.1016/j.pmatsci.2006.08.001.

URL http://www. sciencedirect.com/science/article/pii/S0079642506000417

[4] M. Pratzer, H. Elmers, M. Bode, O. Pietzsch, a. Kubetzka, R. Wiesendanger, Atomic-Scale Magnetic Domain Walls in Quasi-OneDimensional Fe Nanostripes, Physical Review Letters 87 (12) (2001) 127201. doi:10.1103/PhysRevLett.87.127201.

URL http://link.aps.org/doi/10.1103/PhysRevLett.87.127201

[5] P. M. Rørvik, T. Grande, M.-A. Einarsrud, One-dimensional nanostructures of ferroelectric perovskites., Advanced materials (Deerfield Beach, Fla.) 23 (35) (2011) 4007-34. doi:10.1002/adma.201004676.

URL http://www.ncbi.nlm.nih.gov/pubmed/21796684

[6] A. I. Boukai, Y. Bunimovich, J. Tahir-Kheli, J.-K. Yu, W. A. God- 
dard Iii, J. R. Heath, Silicon nanowires as efficient thermoelectric materials, Nature 451 (7175) (2008) 168-171.

[7] T. Zhai, L. Li, X. Wang, X. Fang, Y. Bando, D. Golberg, Recent Developments in One-Dimensional Inorganic Nanostructures for Photodetectors, Advanced Functional Materials 20 (24) (2010) 4233-4248. doi:10.1002/adfm.201001259.

URL http://doi.wiley.com/10.1002/adfm. 201001259

[8] C. Chappert, A. Fert, F. N. Van Dau, The emergence of spin electronics in data storage., Nature materials 6 (11) (2007) 813-23. doi:10.1038/nmat2024.

URL http://www.ncbi.nlm.nih.gov/pubmed/17972936

[9] D. A. Allwood, G. Xiong, C. C. Faulkner, D. Atkinson, D. Petit, R. P. Cowburn, Magnetic domain-wall logic., Science (New York, N.Y.) 309 (5741) (2005) 1688-92. doi:10.1126/science.1108813.

URL http://www.ncbi.nlm.nih.gov/pubmed/16151002

[10] M. Hayashi, L. Thomas, R. Moriya, C. Rettner, S. S. P. Parkin, Currentcontrolled magnetic domain-wall nanowire shift register., Science (New York, N.Y.) 320 (5873) (2008) 209-11. doi:10.1126/science.1154587.

URL http://www.ncbi.nlm.nih.gov/pubmed/18403706

[11] A. Brataas, A. Kent, H. Ohno, Current-induced torques in magnetic materials, Nature materials 11 (1). doi:10.1038/NMAT3311.

URL http://www .nature.com/nmat/journal/v11/n5/abs/nmat3311.html 
[12] D. L. Graham, H. A. Ferreira, P. P. Freitas, Magnetoresistive-based biosensors and biochips., Trends in biotechnology 22 (9) (2004) 455-62. doi:10.1016/j.tibtech.2004.06.006.

URL http://www.ncbi.nlm.nih.gov/pubmed/15331226

[13] H.-T. Huang, T.-R. Ger, Y.-H. Lin, Z.-H. Wei, Single cell detection using a magnetic zigzag nanowire biosensor., Lab on a chip 13 (15) (2013) 3098-104. doi:10.1039/c3lc50457c.

URL http://www.ncbi.nlm.nih.gov/pubmed/23752134

[14] J. S. Jiang, S. D. Bader, Rational design of the exchange-spring permanent magnet., Journal of physics. Condensed matter : an Institute of Physics journal 26 (6) (2014) 064214. doi:10.1088/09538984/26/6/064214.

URL http://www.ncbi.nlm.nih.gov/pubmed/24469386

[15] N. Winkler, J. Leuthold, Y. Lei, G. Wilde, Large-scale highly ordered arrays of freestanding magnetic nanowires, Journal of Materials Chemistry 22 (32) (2012) 16627. doi:10.1039/c2jm33224h.

URL http://xlink.rsc.org/?DOI=c2jm33224h

[16] N. Biziere, C. Gatel, R. Lassalle-Balier, M. C. Clochard, J. E. Wegrowe, E. Snoeck, Imaging the fine structure of a magnetic domain wall in a $\mathrm{Ni}$ nanocylinder., Nano letters 13 (5) (2013) 2053-7. doi:10.1021/nl400317j. URL http://www.pubmedcentral.nih.gov/articlerender.fcgi?artid=3650658\&tool=pm

[17] N. S. Lee, D. S. Chung, I. T. Han, J. H. Kang, Y. S. Choi, H. Y. Kim, S. H. Park, Y. W. Jin, W. K. Yi, M. J. Yun, J. E. Jung, C. J. Lee, Y. , 
S. H. Jo, C. G. Lee, J. M. Kim, Application of carbon nanotubes to field emission displays, Diamond and Related Materials 10 (2) (2001) 265-270. doi:10.1016/S0925-9635(00)00478-7. URL http://www.sciencedirect.com/science/article/B6TWV-42FS2BN-T/2/7bbb5930e4

[18] N. de Jonge, Y. Lamy, K. Schoots, T. H. Oosterkamp, High brightness electron beam from a multi-walled carbon nanotube, Nature 420 (6914) (2002) 393-395. doi:10.1038/nature01233.

URL http://dx.doi.org/10.1038/nature01233

[19] S. Iijima, Helical microtubules of graphitic carbon, Nature 354 (6348) (1991) 56-58. doi:10.1038/354056a0.

URL http://dx.doi.org/10.1038/354056a0

[20] M. Monthioux (Ed.), Carbon Meta-Nanotubes: Synthesis, Properties and Applications, 1st Edition, Wiley, 2011.

[21] R. H. Baughman, A. A. Zakhidov, W. A. d. Heer, Carbon nanotubes-the route toward applications, Science 297 (5582) (2002) 787-792, PMID: 12161643. doi:10.1126/science.1060928.

URL http://www. sciencemag. org/content/297/5582/787

[22] A. V. Eletskii, Carbon nanotube-based electron field emitters, PhysicsUspekhi 53 (9) (2010) 863. doi:10.3367/UFNe.0180.201009a.0897.

URL http://iopscience.iop.org/1063-7869/53/9/R01

[23] F. Houdellier, A. Masseboeuf, M. Monthioux, M. J. Hÿtch, New carbon cone nanotip for use in a highly coherent cold field emission electron microscope, Carbon 50 (5) (2012) 2037-2044. 
doi:10.1016/j.carbon.2012.01.023.

URL http://www.sciencedirect.com/science/article/pii/S0008622312000541

[24] S.-H. Chung, R. D. McMichael, D. T. Pierce, J. Unguris, Phase diagram of magnetic nanodisks measured by scanning electron microscopy with polarization analysis, Physical Review B 81 (2) (2010) 024410. doi:10.1103/PhysRevB.81.024410.

URL http://link.aps.org/doi/10.1103/PhysRevB.81.024410

[25] C. Phatak, A. Petford-Long, O. Heinonen, M. Tanase, M. De Graef, Nanoscale structure of the magnetic induction at monopole defects in artificial spin-ice lattices, Physical Review B 83 (17) (2011) 174431. doi:10.1103/PhysRevB.83.174431.

URL http://link.aps.org/doi/10.1103/PhysRevB.83.174431

[26] C. Gatel, a. Lubk, G. Pozzi, E. Snoeck, M. Hÿtch, Counting Elementary Charges on Nanoparticles by Electron Holography, Physical Review Letters 111 (2) (2013) 025501. doi:10.1103/PhysRevLett.111.025501.

URL http://link.aps.org/doi/10.1103/PhysRevLett.111.025501

[27] D. Shindo, Y. Murakami, Electron holography study of electric field variations., Journal of electron microscopy 60 Suppl 1 (Supplement 1) (2011) S225-37. doi:10.1093/jmicro/dfr017.

URL http://www.ncbi.nlm.nih.gov/pubmed/21844592

[28] J. Cumings, A. Zettl, M. R. McCartney, J. C. H. Spence, Electron holography of field-emitting carbon nanotubes, Physical Review Letters 
88 (5) (2002) 056804. doi:10.1103/PhysRevLett.88.056804.

URL http://link.aps.org/doi/10.1103/PhysRevLett.88.056804

[29] Y. Aharonov, D. Bohm, Significance of electromagnetic potentials in the quantum theory, Physical Review 115 (3) (1959) 485-491.

[30] A. Lubk, D. Wolf, P. Simon, C. Wang, S. Sturm, C. Felser, Nanoscale three-dimensional reconstruction of electric and magnetic stray fields around nanowires, Applied Physics Letters 105 (17) (2014) 173110. doi:10.1063/1.4900826.

URL http://scitation.aip.org/content/aip/journal/apl/105/17/10.1063/1.4900826

[31] T. Tanigaki, Y. Takahashi, T. Shimakura, T. Akashi, R. Tsuneta, A. Sugawara, D. Shindo, Three-Dimensional Observation of Magnetic Vortex Cores in Stacked Ferromagnetic Discs., Nano lettersdoi:10.1021/nl504473a.

URL http://www.ncbi.nlm.nih.gov/pubmed/25594686

[32] D. Wolf, L. A. Rodriguez, A. Béché, E. Javon, L. Serrano, C. Magen, C. Gatel, A. Lubk, H. Lichte, S. Bals, G. Van Tendeloo, A. Fernández-Pacheco, J. M. De Teresa, E. Snoeck, 3D Magnetic Induction Maps of Nanoscale Materials Revealed by Electron Holographic Tomography, Chemistry of Materials 27 (19) (2015) 6771-6778. doi:10.1021/acs.chemmater.5b02723.

[33] R. E. Dunin-Borkowski, T. Kasama, A. Wei, S. L. Tripp, M. J. Hÿtch, E. Snoeck, R. J. Harrison, A. Putnis, Off-axis electron holography of magnetic nanowires and chains, rings, and planar arrays of magnetic 
nanoparticles, Microscopy research and technique 64 (5-6) (2004) 390402.

[34] J. Tatum, W. Jaworski, A solution of abel's equation, Journal of Quantitative Spectroscopy and Radiative Transfer 38 (4) (1987) 319-322. URL http://www.sciencedirect.com/science/article/pii/0022407387900768

[35] J. D. Jackson, Classical electrodynamics, 3rd Edition, Wiley, New York, 1999.

URL http://www . loc.gov/catdir/description/wiley033/97046873.html

[36] M. De Graef, N. Nuhfer, M. McCartney, Phase contrast of spherical magnetic particles, Journal of Microscopy 194 (1) (1999) 84.

[37] S. Ma, H. Gao, L. Wu, Modified Fourier-Hankel method based on analysis of errors in Abel inversion using Fourier transform techniques., Applied optics 47 (9) (2008) 1350-7.

URL http://www.ncbi.nlm.nih.gov/pubmed/18709083

[38] A. Savitzky, M. Golay, Smoothing and Differentiation of Data by Simplified Least Squares Procedures, Analytical chemistry 36 (8) (1964) $1627-1639$.

URL http://pubs . acs .org/doi/abs/10.1021/ac60214a047

[39] L. de Knoop, F. Houdellier, C. Gatel, A. Masseboeuf, M. Monthioux, M. Hÿtch, Determining the work function of a carbon-cone coldfield emitter by in situ electron holography, Micron 63 (2014) 2-8. doi:10.1016/j.micron.2014.03.005. URL http://www.sciencedirect.com/science/article/pii/S0968432814000547 
[40] J.-M. Bonard, M. Croci, I. Arfaoui, O. Noury, D. Sarangi, A. Châtelain, Can we reliably estimate the emission field and field enhancement factor of carbon nanotube film field emitters?, Diamond and Related Materials 11 (3-6) (2002) 763 - 768. doi:http://dx.doi.org/10.1016/S09259635(01)00541-6.

URL http://www.sciencedirect.com/science/article/pii/S0925963501005416

[41] J. F. Einsle, C. Gatel, A. Masseboeuf, R. Cours, M. A. Bashir, M. Gubbins, R. M. Bowman, E. Snoeck, In situ electron holography of the dynamic magnetic field emanating from a hard-disk drive writer, Nano Research 8 (4) (2014) 1241-1249. doi:10.1007/s12274-014-0610-0.

URL http://dx.doi.org/10.1007/s12274-014-0610-0

[42] M. Beleggia, T. Kasama, D. J. Larson, T. F. Kelly, R. E. DuninBorkowski, G. Pozzi, Towards quantitative off-axis electron holographic mapping of the electric field around the tip of a sharp biased metallic needle, Journal of Applied Physics 116 (2). doi:10.1063/1.4887448.

URL http://dx.doi.org/10.1063/1.4887448 


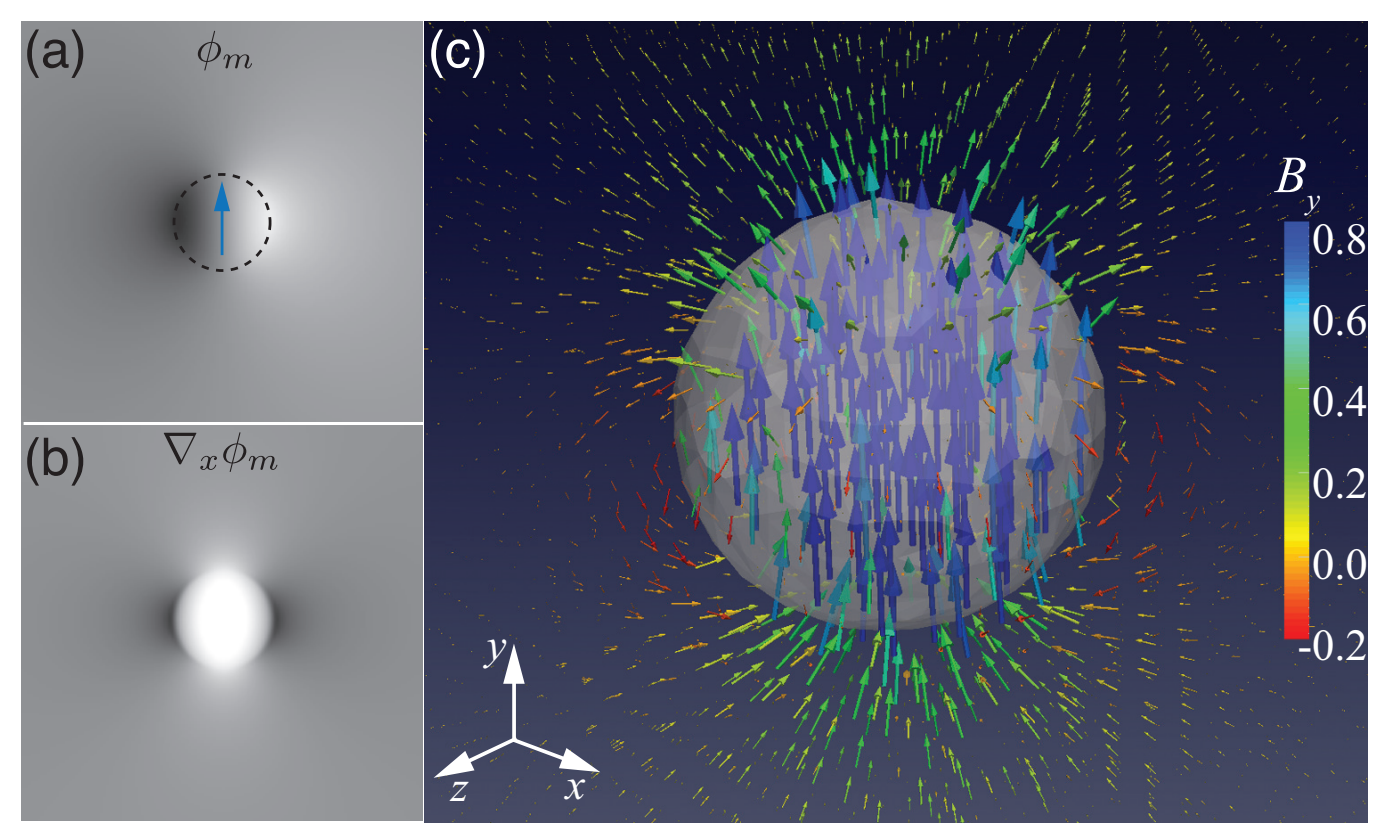

Figure 1: (a) shows the magnetic phase shift image of a uniformly magnetized spherical nanoparticle, indicated by the dashed line, and magnetization vector is indicated by the blue arrow, (b) the derivative of the magnetic phase shift with respect to $x$ showing the symmetry about the vertical axis of the image, and (c) the 3D reconstructed magnetic induction inside and outside the spherical particle. The color bar shows the magnitude of the $y$ component of the magnetic induction, $B_{y}$, in T. 
(a)

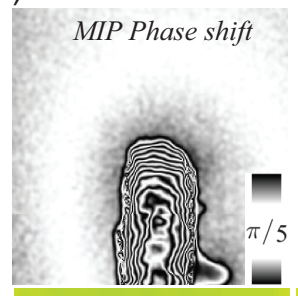

(c) $B_{y}$ (projection) (b)

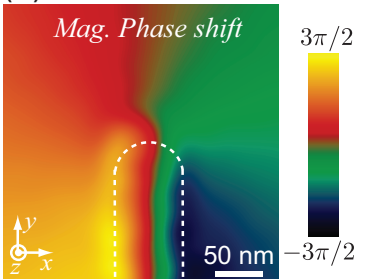

(d) $B_{y}(x-y$ plane $)$

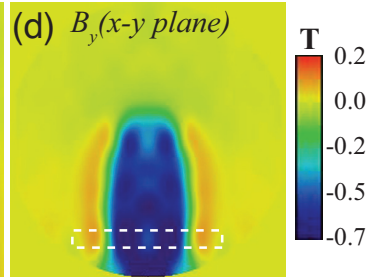

(e) $3 D$ Vector field map

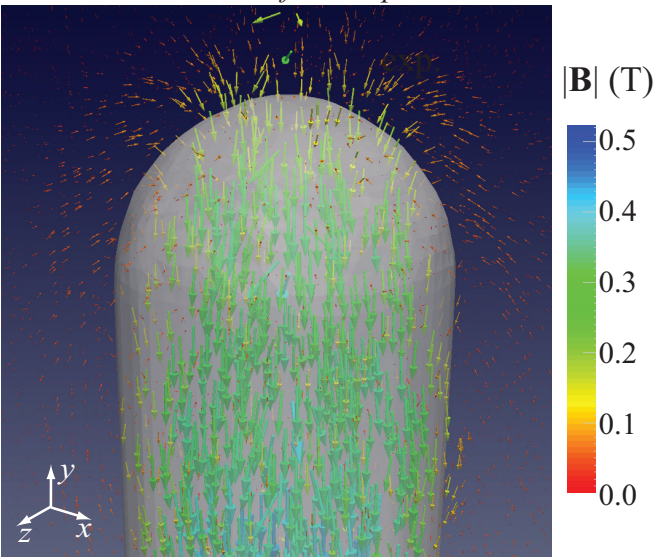

Figure 2: (a) shows the electrostatic contribution to the phase shift displayed as $\cos (5 \Delta \phi)$ of the nickel nanowire. The associated scale displays the amount of $\phi_{e}$ between dark lines (b) shows the magnetic contribution to the phase shift $\left(\phi_{m}\right)$. (c) shows the $B_{y}$ component calculated from the gradient of magnetic phase shift, and (d) shows the $B_{y}$ component in the $x-y$ plane in the middle of the nanowire. The color represents the strength of magnetic induction indicated by the colorbar. (e) shows the $3 \mathrm{D}$ vector plot of the reconstructed magnetic induction from the nanowire. The vectors are colored according to the $y$ component of the induction indicated by the color bar (in units of $\mathrm{T}$ ). 
(a) Magnetic Field in various planes
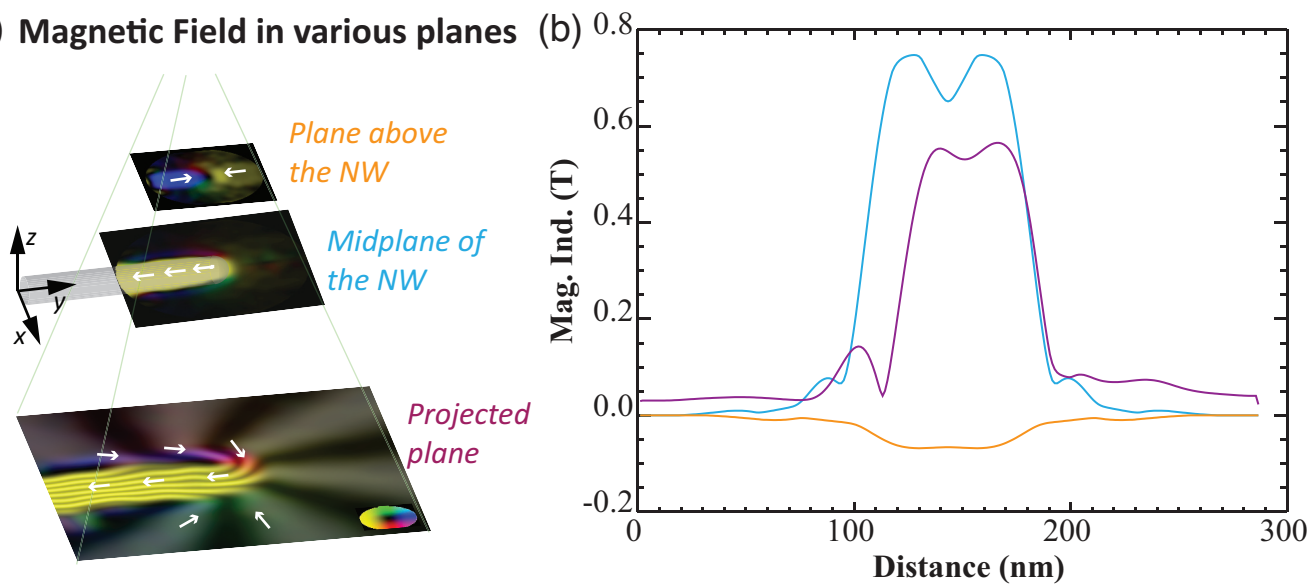

Figure 3: (a) shows a schematic to illustrate the advantage of our method by showing the magnetic induction that can be measured at various locations in 3D as opposed to the projected magnetic induction, and (b) shows the quantitative comparison of the magnetic induction at various locations represented in (a) from the white dashed region shown in Figure 2(d). 

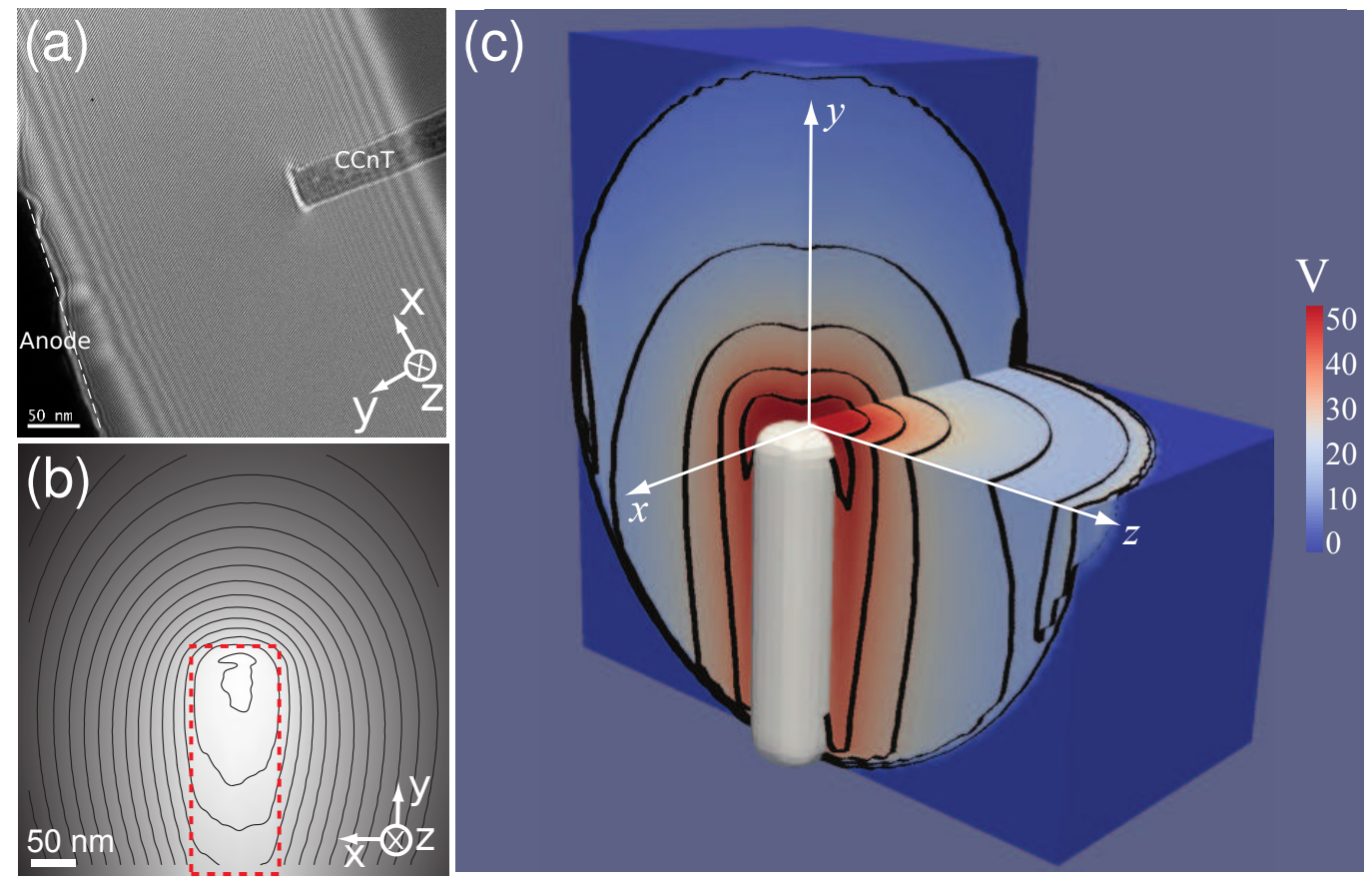

Figure 4: (a) shows the as acquired interference hologram, which shows the positions of the $\mathrm{CCnT}$ and the anode. (b) shows the experimentally measured phase shift from a carbon cone nanotip (CCnT) under an applied bias of $60 \mathrm{~V}$, (c) shows the reconstructed 3D distribution of potential (in V) around the CCnT along with contours drawn every 10 V. 

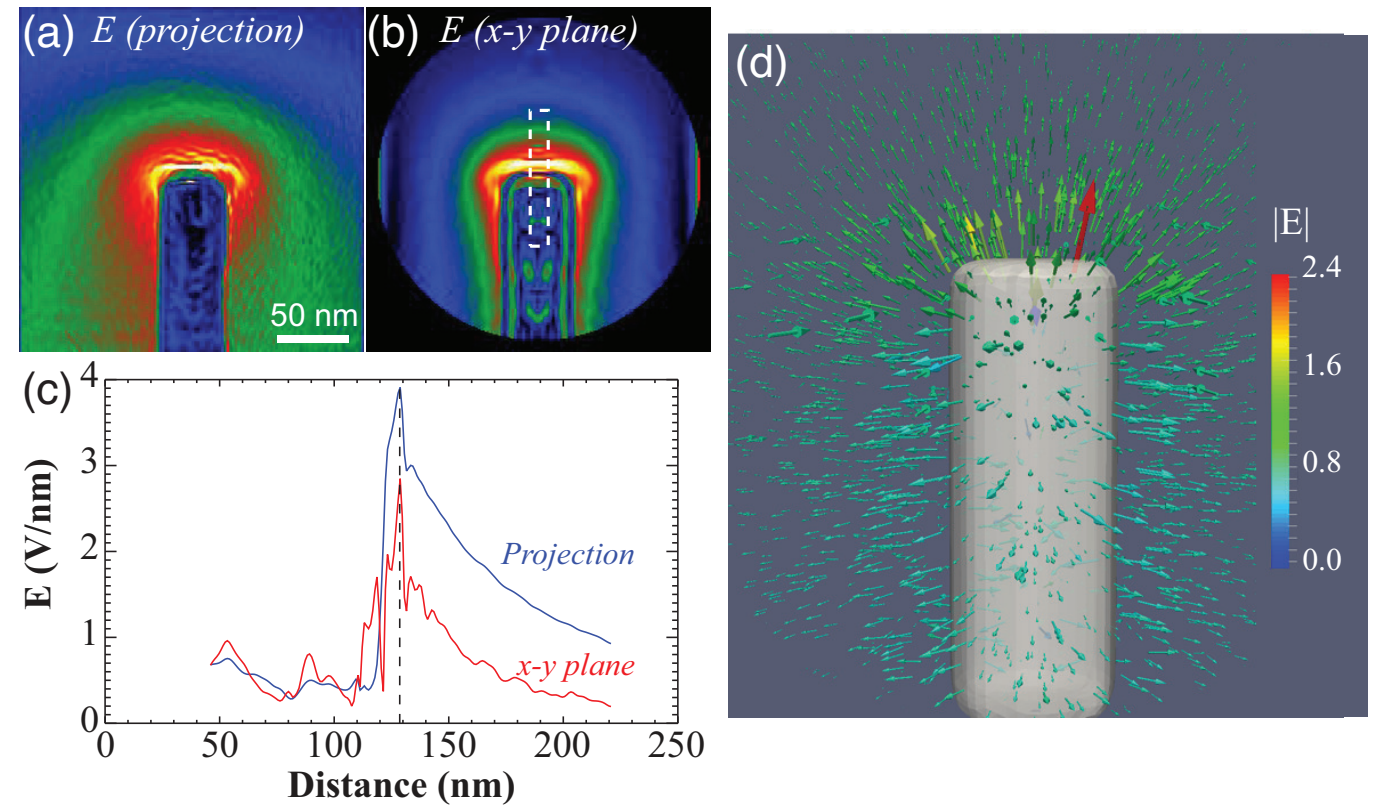

Figure 5: (a) shows reconstructed electric field in the projection plane computed from gradient of the phase shift, and (b) shows the reconstructed electric field in the $x-y$ plane in the middle of the CCnT. (c) shows the quantitative comparison of the reconstructed electric field in the projection plane and the $x-y$ plane in the middle of the CCnT. (d) shows the $3 \mathrm{D}$ vectorial view of the reconstructed electric field (in $\mathrm{V} / \mathrm{nm}$ ) around the CCnT. 